\title{
A Preliminary Study of Radiometeorological Effects on Beyond-Horizon Propagation
}

\author{
F. Ikegami ${ }^{1}$
}

(October 22, 1959)

\begin{abstract}
A study was made of American and Japanese radiometeorological data in order to suggest the dominant factors in propagation beyond the horizon. The diurnal variability of radio field strengths seems to be sensitive to the crossover height of rays tangent to the radio horizon and disappears for crossover heights greater than about 500 meters. High hourly median field strengths were observed in Japan corresponding to the existence of a marked refractive index discontinuity layer in a common volume of two antenna beams. The results of these experiments suggest that laminar structures of the atmosphere play an important role in beyond-horizon radio propagation.
\end{abstract}

\section{Introduction}

Detailed investigations have established the correlations between monthly median values of basic transmission loss and radiometeorological factors. These correlations have been successfully applied to design practical communication links utilizing the surface refractivity or the lapse rate of refractivity with height in the first kilometer above the earth's surface $\left[\begin{array}{llllll}1 & 2 & 3 & 4\end{array}{ }^{2}\right.$ The next stage of research requires the estimation of the variation of transmission loss within a month in order to determine the nature of the fading in more detail.

Several theories have been proposed for the explanation of low-transmission loss beyend the horizon unexpected by classic diffraction theory; for example, scattering [5], partial reflection in the tapered atmosphere [6], diffraction [7], reflection at multiple layers [8], reflection at an elevated layer, normal refraction, and superrefraction. Usually the first four stand for lower signal strength, while the last three, for the higher. None of them may be consistent for actually observed propagation phenomena because the observed field is apparently dependent upon various meteorological structures of the atmosphere with different modes of propagation.

However, the solution of some engineering problems requires a knowledge of the main factors which effectively predominate in actual propagation phenomena rather than the determination of the propagation mechanism to which the lowest field intensity beyond the horizon (so-called "scattered" level) is attributed.

This paper describes a preliminary study of the meteorological effects on beyond-horizon propagation, based upon the experimental results obtained by NBS and the Electrical Communication Lab. of Nippon Telegraph and Telephone Corp., Japan

\footnotetext{
1 Electrical Communication Lab., Nippon Telegraph and Telephone Corp., Tokyo, Japan, on leave as a United Nations Technical Assistance Fellow.

2 Flgures in brackets indicate the literature references at the end of this paper.
}

(ECL), and presents two aspects that should be taken into consideration for further studies towards practical application. The first aspect concerns the well-known diurnal variation of transmission loss over land; the second concerns low elevated layers that appear from time to time in the atmosphere.

Some considerations of the experimental relations between radio and meteorological data are made and suggestions offered for further studies in this field.

\section{Diurnal Variation of Hourly Median Transmission Loss Beyond the Horizon}

\subsection{Description of Experimental Results (NBS)}

The results of the extensive propagation experiments conducted by NBS show that the transmission loss to points near and just beyond the horizon is subjected to a systematic diurnal change, and that the diurnal variation disappears far beyond the horizon. The dependence of diurnal variation on angular distance has been reported in detail $[1,9]$. We will examine the diurnal variation from another standpoint. In order to compare diurnal variations for various propagation paths, the period of August 1952 was chosen mainly because measurements of transmission loss were carried out simultaneously or in similar meteorological conditions for the waves radiated at Cheyenne Mt., Camp Carson, and Pikes Peak. The lengths of the periods of data used in this analysis were not always long enough to completely express all diurnal characteristics, since some are even of insufficient length for analysis. All data were used here, however, in order to complement the shortage of varieties in propagation paths.

In figure 1 is shown the mean diurnal variation of hourly median transmission loss for various propagation paths in terms of increasing height of crossover point of the two horizons above the ground. The height of the cross-over point is used in this paper instead of angular distance in order to discuss its connection with meteorological effects on diurnal 

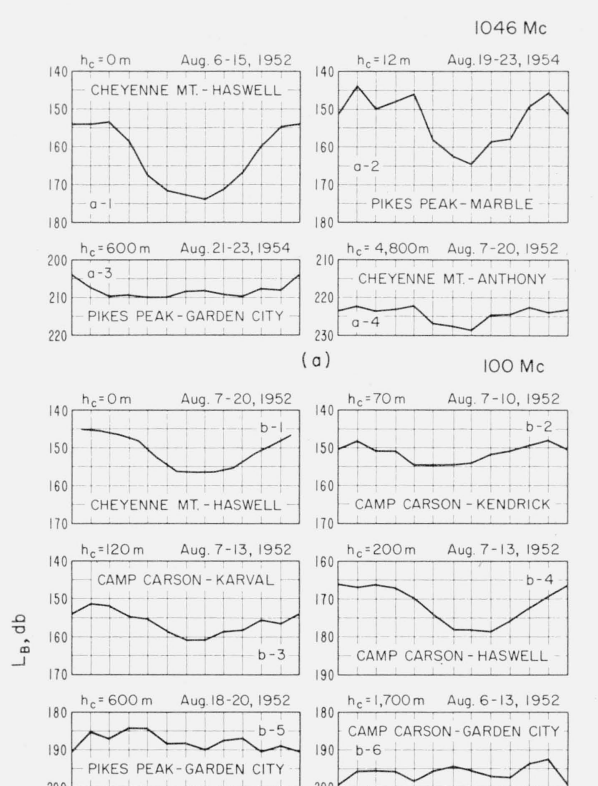

200 PIKES PEAK - GARDEN CITY
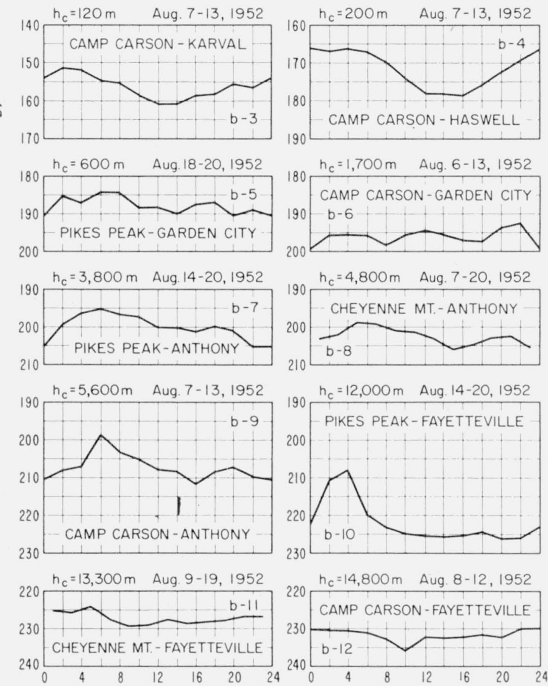

LOCAL TIME

(b)

Figure 1(a) and 1(b). Mean diurnal variations of hourly median transmission loss.

variation of transmission loss. The height of the crossover point, of course, corresponds to angular distance with a one-to-one correspondence, if a spherical smooth earth is assumed. It is easily seen from figure 1 that systematic diurnal characteristics rapidly disappear with increasing height of the crossover point, and that amplitudes of systematic diurnal variation depend on radiofrequency and have a maximum just bevond the horizon, as pointed out in reference [1]. We will see that the unusual increases of field strength seen on paths between Pikes Peak and Fayetteville and between Camp Carson and Anthony do not stand for systematic diurnal characteristics but rather are due to unusual signal enhancement that took place once on each path during the test period.

We now wish to examine the dependence of systematic diurnal characteristics of the transmission loss upon the height of the crossover point. The amplitude of the diurnal variation itself has an important meaning in practical engineering and should be analyzed in a further stage of research based upon a large quantity of data obtained on various propagation paths and in various seasons, and/or upon the propagation mechanism, if clarified, in relation with pertinent radiometeorological factors.

Several methods might be available to express the importance of systematic diurnal characteristics in the total variation of transmission loss. A rough but simple method is applied here as a trial. We assume that the total range $\Delta L$ of the hourly median transmission losses consists of two components, $\Delta L_{d}$ and $\Delta L_{n}$, the former being subjected to systematic diurnal variation and the latter to nonsystematic variation. Then

$$
V_{d}=\frac{\Delta L_{d}}{\Delta L}=\frac{\Delta L_{d}}{\Delta L_{d}+\Delta L_{n}}
$$

represents the importance of diurnal variation in the total variation, which may be temporarily referred to as "diurnal variability" hereafter. Noting that maximum and minimum signal strengths are $e x-$ pected at 02 and $14 \mathrm{hr}$ of the day, respectively, in an ordinary diurnal pattern, and considering that radiosonde observation data at Denver are available at these times, the difference of transmission loss in decibels at 02 and $14 \mathrm{hr}$ divided by the maximum variation of transmission loss observed during the test period is used as an expression of diurnal variability.

That is:

$$
V_{d}=\frac{\left(\bar{L}_{B}\right)_{14}-\left(\bar{L}_{B}\right)_{02}}{\left(L_{B}\right)_{\max }-\left(L_{B}\right)_{\min }}
$$

where

$$
V_{d}=\text { diurnal variability }(\leq 1),
$$

$\left(\bar{L}_{B}\right)_{14}=$ mean of hourly median transmission losses at $14^{\mathrm{h}}$,

$\left(\bar{L}_{B}\right)_{02}=$ mean of hourly median transmission losses at $02^{\mathrm{h}}$,

$\left(L_{B}\right)_{\max }=$ maximum hourly median basic transmission loss during the test period,

$\left(L_{B}\right)_{\mathrm{min}}=$ minimum hourly median basic transmission loss during the test period.

This value is not an expression of the absolute importance of diurnal variation but is merely a relative one. Comparison of diurnal variability, therefore, should be made for the same or similar meteorological conditions. A large value of $V_{d}$ means that a large portion of the total variation is caused by factors subjected to a systematic diumal characteristic, and vice versa.

The variation of diurnal variability with crossover height is shown in figure 2 for the propagation paths mentioned above, as well as for that between Cedar Rapids and Quincy [9]. Except for one point of the Cheyenne Mt.-Anthony path at 1,046 Me, plotted points show a clear tendency for $V_{d}$ to be larger the lower the crossover height, $h_{c}$. Further, $V_{d}$ decreases rapidly as $h_{c}$ increases. The value of $V_{d}$ seems 10 


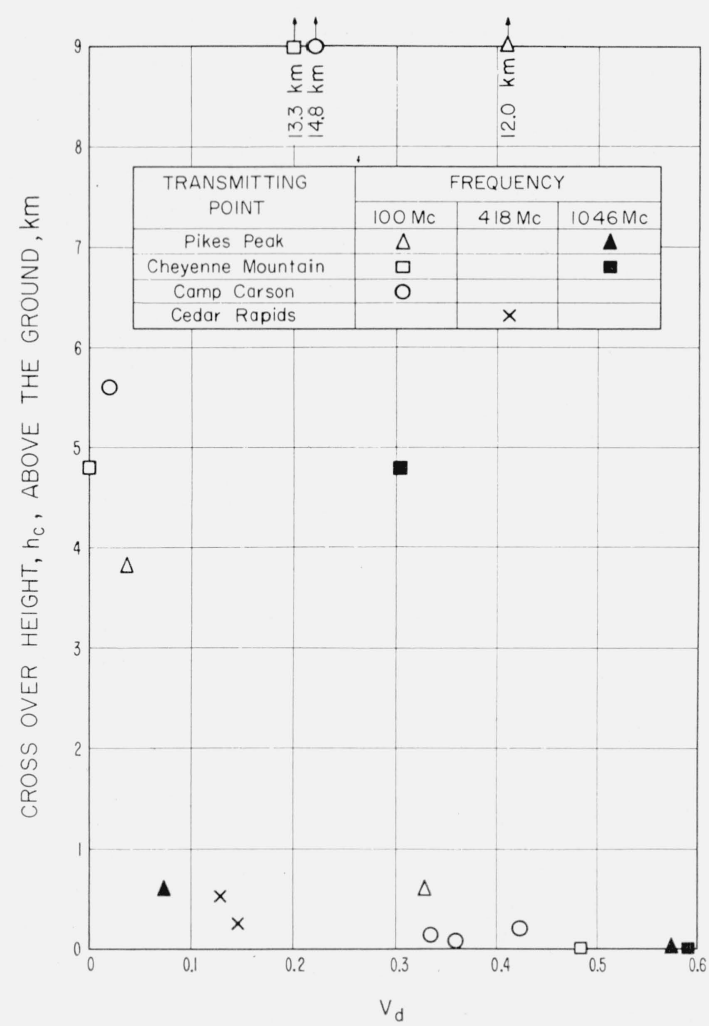

Figure 2. Variation of diurnal variability with crossover height.

increase at extreme heights of crossover point. These 6 xtreme points are all for Fayetteville, and correspond to the case described above when an unusual increase in field strength occurred once during a short test period. This result indicates that a systematic diurnal variation appears when the crossover height is located below about $500 \mathrm{~m}$ above the ground and diminishes almost completely above about $1,000 \mathrm{~m}$.

It is interesting to note that a similar tendency of $V_{d}$ is seen regardless of the height of the transmitters; for example, the transmitter height was very much larger on the Pikes Peak path than on the Camp Carson and Cedar Rapids paths. This shows the significance of crossover height, rather than either that of distance or of antenna height, in the expression of diurnal variation of transmission loss.

\subsection{Considerations of Diurnal Variation}

Out of several modes of propagation beyond the horizon, a combination of diffracted and scattered field seems a most probable explanation of diurnal variation. However, this fails to explain the large amplitude of diurnal variation by using the ordinary linear $N$-profile concept [1].

Another interesting phenomenon should be pointed out. Field strengths recorded within an hour on both the Camp Carson-Haswell and Cedar Rapids-Quincy paths are frequently subjected to Ray- leigh distributed fading. This even occurs during the night when median field intensities are higher by 10 or more decibels than in the daytime. We find a difficulty in trying to explain this phenomenon by means of an ordinary combination of diffraction and scattering modes with only refraction in the lowest part of the atmosphere superimposed. The exact answer for this question is very difficult to obtain so far, as measurements are not yet available for the fine structure of the lower atmosphere.

At this point we are reminded of the dependence of diurnal variability on crossover height. It is well known that the lower part of the atmosphere is deformed by solar radiation and nocturnal cooling with a distinguished diurnal cycle, and very much has been discussed about diurnal variation in the structure of the lower atmosphere. It is interesting to note that diurnal variation of the atmosphere is limited to the lowest part, ranging from the earth's surface up to $500 \mathrm{~m}$ but perhaps not higher than $1,000 \mathrm{~m}$ above the ground. For example, according to the extensive observations at Larkhill and Downham Market [10], mean heights of temperature inversion layers are 200 to $300 \mathrm{~m}$ depending upon season and time of the day. Similar results were obtained in radiometeorological observations performed by ECL [11] utilizing a $300 \mathrm{~m}$ high tower. This latter experiment showed that temperature inversion layers extending up to 200 - or $300-\mathrm{m}$ high commonly displaved a diurnal cycle, and the gradient of refractive index versus height also showed a remarkable diurnal variation. Since the exact mechanism of radio propagation has not been clarified yet, no specific meteorological factor could be discussed here with particular significance. However, the above results may be compared with figure 3 , which shows the diurnal variation of $N$-gradient at various heights above the ground observed in Denver during the radio test period in August 1952. Differences of $N$-gradient at $02^{\mathrm{h}}$ and $14^{\mathrm{h}}$, plotted in figure 4 against height, indicate that diurnal variation in the refractive index structure of the atmosphere rapidly decreases with height and disappears above about $500 \mathrm{~m}$.

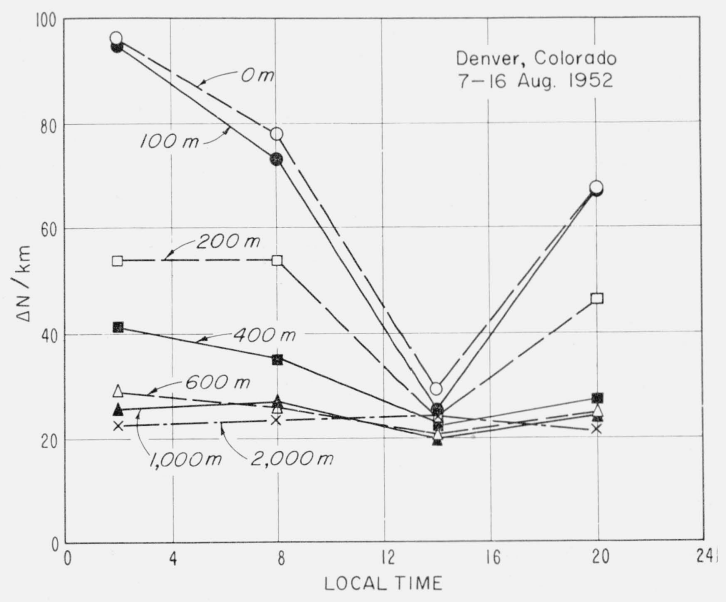

Figure 3. Diurnal variations of the gradient of refractivity at various heights above the ground. 


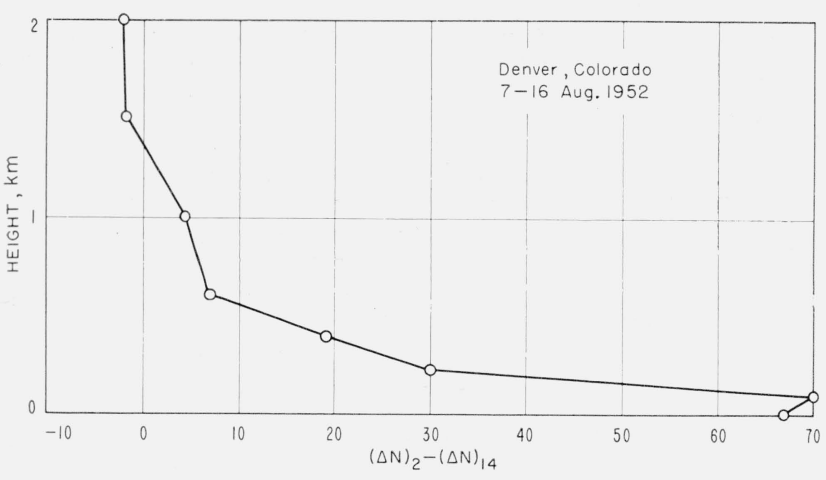

Figure 4. Diurnal range of $\Delta \mathrm{N} / \mathrm{km}$ versus height above the ground.

Though a comparison of figures 2 and 4 is not based on any strict physical reason, the similarity shown in these figures might suggest the large contribution of the atmosphere near the crossover point of the path to propagation beyond the horizon. In fact, the difficulty may be reduced by assuming that the field strength beyond the horizon is largely dependent upon the refractive index structure of the atmosphere, for instance, laminar structure in the lower part of the common volume of two antenna beams, considering that the lowest part of the atmosphere may contribute to emphasize it by lowering the crossover height.

\section{Effects of an Elevated Layer in Beyond- Horizon Propagation}

\subsection{Experimental Results (ECL)}

The Radio Wave Propagation Section of Electrical Communication Lab., N.T. \& T., Japan, conducted a series of beyond-horizon oversea propagation tests from the fall of 1957 until the summer of 1958 [12]. The map and terrain profile of propagation paths of Miyazaki-Muroto and Miyazaki-Wakayama are shown in figures 5 and 6 . In the test of May 1958,

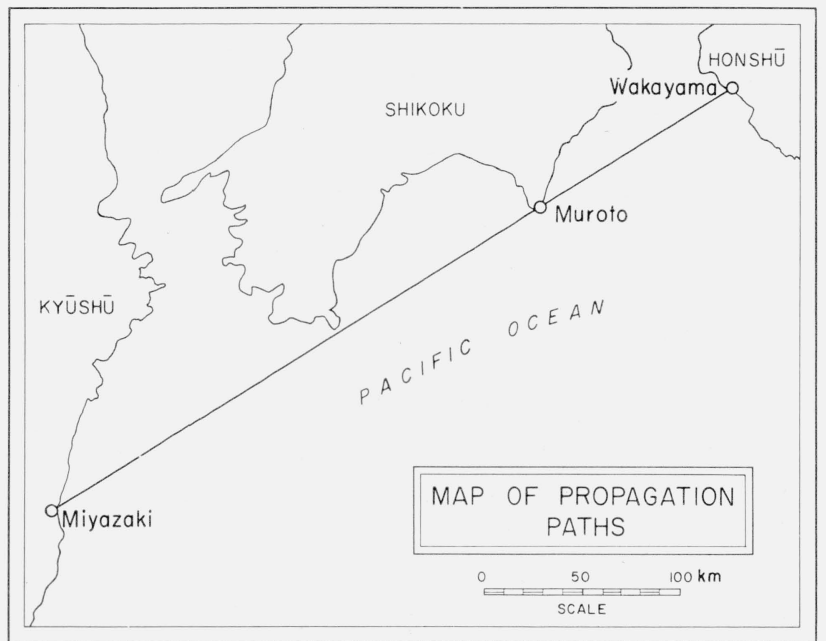

Figure 5. Map of propagation paths.
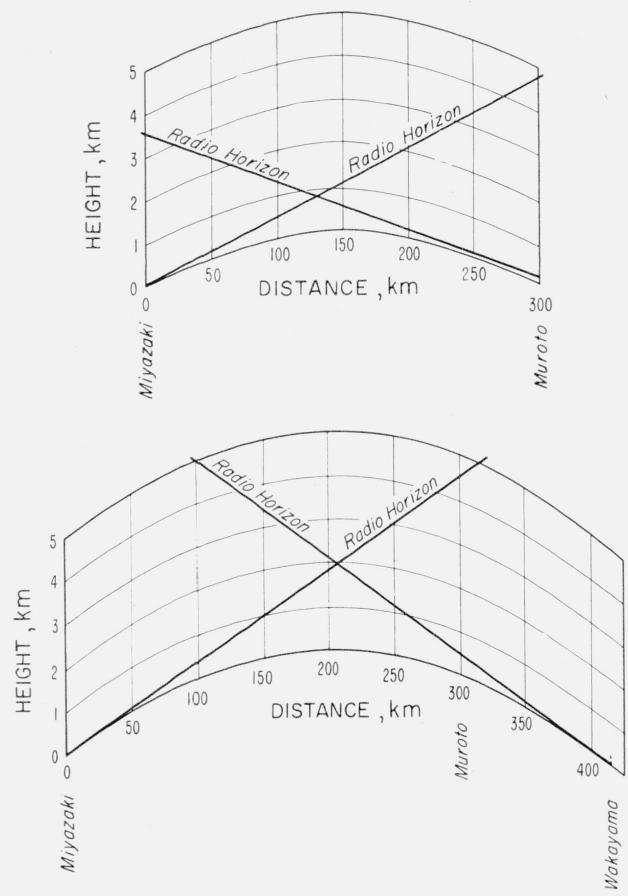

Figure 6. Profiles of propagation paths.

radiosonde observations were performed at Muroto four times a day in order to analyze the effects of radiometeorological conditions on radio propagation.

The 1,300- and 3,000-Mc hourly median basic transmission losses are used in the present analysis. Calculations of refractive index were made at each transition point of temperature code of radiosonde which covered the height interval from 0 to 5,000 or to $7,000 \mathrm{~m}$.

In figure 7 is shown the variation of median basic transmission loss for both frequencies and for both propagation paths, compared with radiometeorological factors; they are: the gradient of refractive index in every $500 \mathrm{~m}$ classified into three grades as shown in the figure, and relative humidity at 1,000 and $3,000 \mathrm{~m}$ above sea level. It is seen in this figure that high signal strength is accompanied by steep $N$-gradient at a considerable height for both frequencies and for both propagation paths. These layers are mainly caused by an extremely dry airmass in a high pressure area lying above a humid airmass. It is shown by the relative humidity curves that elevated layers appear when a large difference of relative humidity occurs between 1,000 and $3,000 \mathrm{~m}$. In this season of the year, high pressure areas periodically traverse Japan from west to east. In response to the traverse of high pressure areas, median transmission loss presents a similar variation with a period of about 4 days. Systematic diurnal variation of transmission loss was not observed at all. Variations seem to be dependent largely on the property of the airmass covering the propagation path.

Correlation coefficients were calculated between transmission loss and $N$-gradient for two paths and 

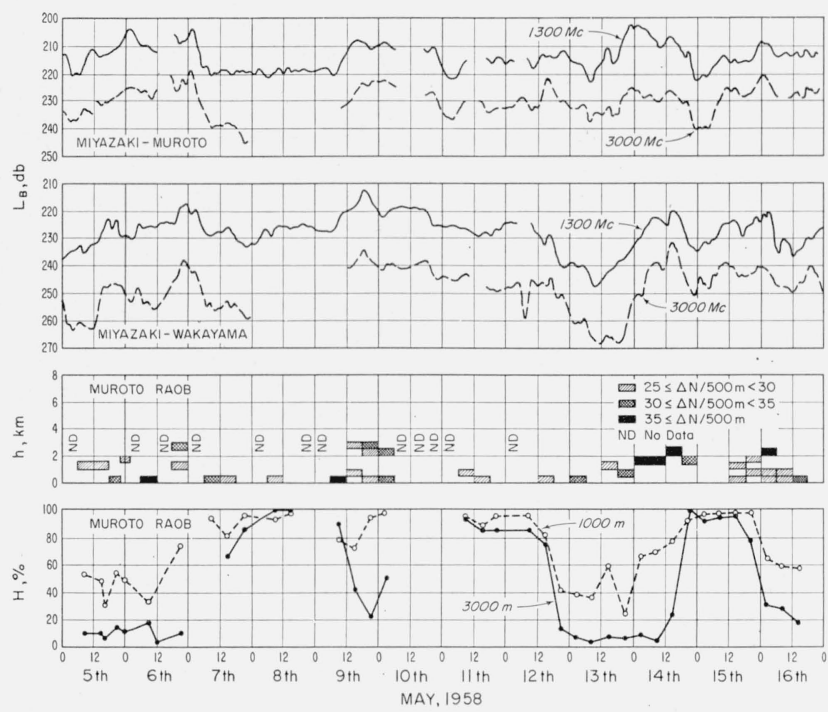

Figure 7. Comparison of radio and meteorological data.

For this figure only, $H$ refers to the relative humidity in percent.

for the frequency of $3,000 \mathrm{Mc}$. In this case, the maximum value of $\Delta N / 500 \mathrm{~m}$ in the height range higher than a height $H$ was correlated with transmission loss, considering that all layers located higher than $H$ are concerned with the received field strength. Correlation coefficients calculated for various values of $H$ are plotted against $H$ in figure 8 . It is interesting to note that the maximum value of the correlation coefficient is observed near the crossover height of each propagation path, and that

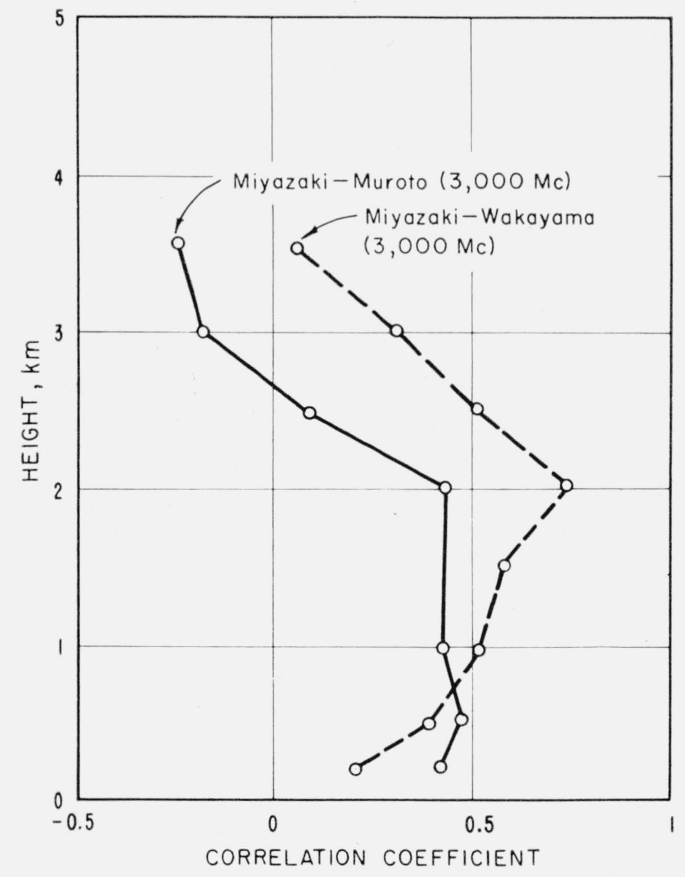

Figure 8. Variation of correlation coefficient between median path loss and $(\Delta \mathrm{N} / 500 \mathrm{~m})_{\max }$ with height. larger values of correlation coefficient are obtained on the Miyazaki-Wakayama path, the crossover point of which is closest to the radiosonde observation point.

No significant correlation was found, on the other hand, between transmission loss and $N$-gradients in the lowest 50,200 , and $1,000 \mathrm{~m}$ of the atmosphere.

A comparison with surface weather maps showed that high field strengths and elevated layers both accompanied high pressure areas, as stated above. A typical case, observed between the 12 th and 15 th of May when a high pressure area passed over the propagation paths, was analyzed in order to present the effect of a layer in more detail. Figure 9 shows a time cross section of relative humidity up to $7,000 \mathrm{~m}$ above sea level compared with variations in transmission loss during the same period. Equi-relative humidity contour lines are drawn in the figure for every 10 percent; an area of closely spaced lines denotes the existence of a layer with steep $N$-gradient. An extremely dry airmass, with relative humidity less than 10 percent, perhaps due to subsidence in the rear of the high pressure area, appeared on the afternoon of the 12th of May, producing a marked elevated discontinuity layer between 1,000 and 2,500
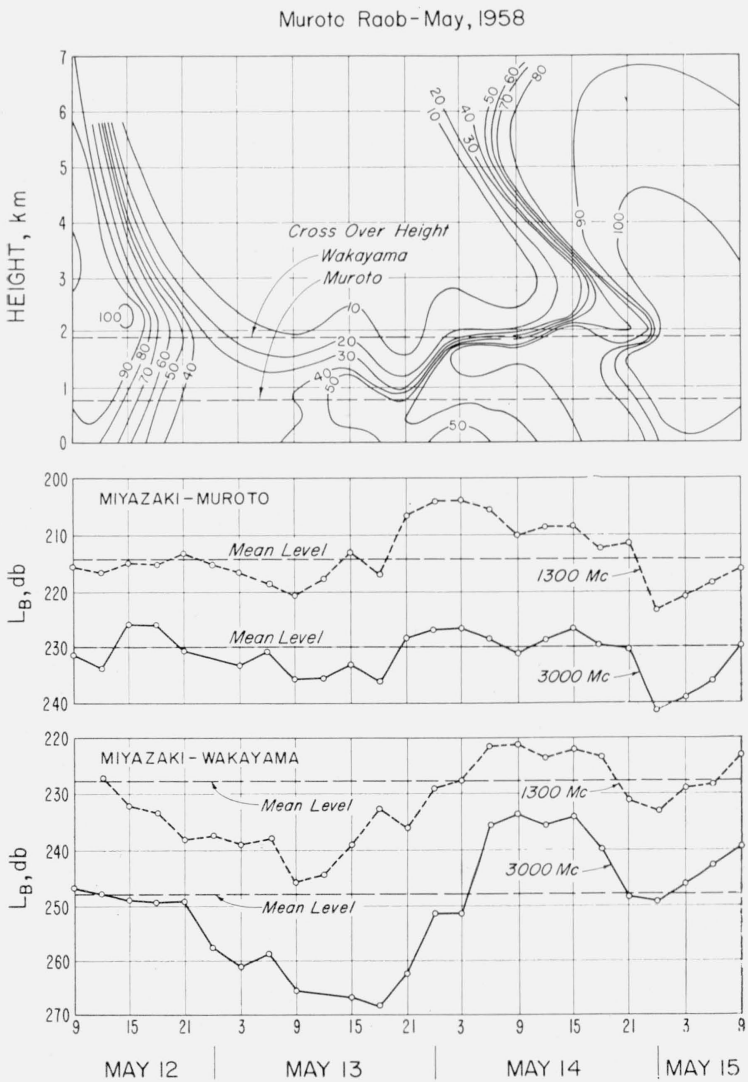

Figure 9. The cross section of relative humidity in a high pressure area compared with transmission loss variation. 
$m$ by the afternoon of the 14th. Comparing the transmission loss curves, shown in the lower part of the figure, with the variation of this layer, it is seen that high field strength started on the Miyazaki-Muroto path coincidently with the layer appearance at $21^{\mathrm{h}}$ on the 13 th and ended when it disappeared at $21^{\mathrm{h}}$ on the 14th. Further, high signal strength started on the Miyazaki-Wakayama path when the layer reached up to the height of crossover point of the path at $06^{\mathrm{h}}$ on the 14 th. This shows that an appearance of an elevated layer higher than the crossover height produces a high field strength at the receiving point. It is also interesting to note that no change was found in the distribution of short term fading, which was represented by a Rayleigh distribution, during the whole process from the lowest through the highest field strength. This fact indicates that even a marked elevated layer does not act as a specular reflection plane, but produces many rays, numbering three or more, by finer structure of the layer.

As far as this propagation test is concerned the effects of the elevated layer played the most important role in the variation of median transmission loss within the month, occasional discrepancies being seen between them, perhaps due to other meteorological factors.

\subsection{Experimental Results (NBS)}

This section describes an attempt to determine relations between transmission loss and the presence of elevated layers, using experimental data obtained by NBS. Radiosonde data observed at Denver four times a day are used. By comparison, it was found that the effect of an elevated layer was not apparently distinguishable during this test period, because it was masked by more remarkable diurnal variations in the lower atmosphere. Two typical cases in August 1952 are shown in figures 10 and 11 , when extreme enhancements in field strength were observed far beyond the horizon. In the first case (fig. 10), a marked discontinuity layer lies near the crossover height of the Pikes Peak-Anthony path. However, it is difficult to ascertain the effect of this laver because of a superrefractive layer observed simultaneously in the lowest part of the atmosphere. The fact that a similar enhancement occurs on the Pikes Peak-Fayetteville path, the crossover height of which is as high as $12,000 \mathrm{~m}$ above the ground, may emphasize the importance of superrefraction.

In the second case (fig. 11), we may be forced to attribute the enhancement occurring on the Camp Carson-Garden City path at $12^{\mathrm{h}}$ to $14^{\mathrm{h}}$ on the 11 th to the layer observed near the height of crossover point (about 1,700 m), while we find a similar difficulty as in the first case (fig. 10) for the enhancements observed at Garden City and Anthony in the morning on the 12th. Further investigations would be necessary with careful treatments of lower superrefractive layers.

\subsection{Considerations on Contribution of Layers ot Propagation Far Beyond the Horizon}

In the experiments by ECL, we found that elevated layers observed by radiosonde were the main factor in propagation far beyond the horizon. The lowest field level, which is expected in a linear or a tapered atmosphere, might be determined by scattering, internal reflection, or diffraction. However, this idealized type of the atmosphere was rarely observed in practice, the actual profile condition being characterized by sudden changes in the first derivative of $N$-profile. We could expect more and finer changes in the atmosphere that were missed by radiosonde because of its insufficient accuracy. Field strength far beyond the horizon seems to be largely contributed by laminar structures in the atmospheric refractive index, even though the lower or the lowest level might be determined by other modes of propagation.

In case of the experiments by NBS, on the other hand, we could not find so apparent effects as in the former case, mainly because of radiosonde data which were available only at significant levels and also be-
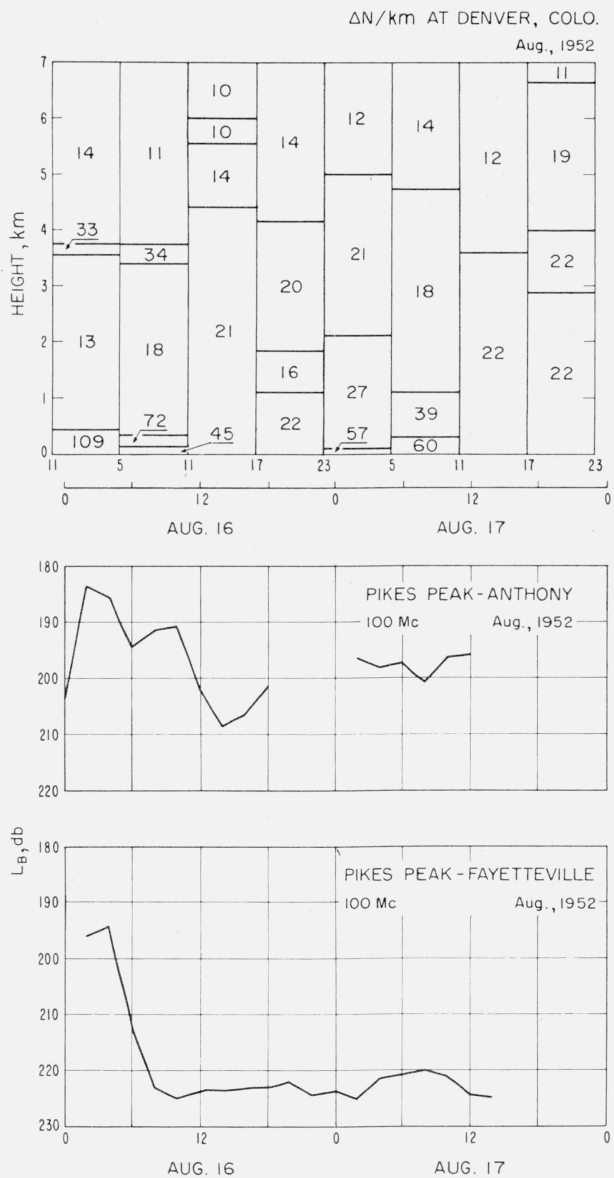

Figure 10. Comparison of transmission loss with the gradient of refractive index of the atmosphere. 

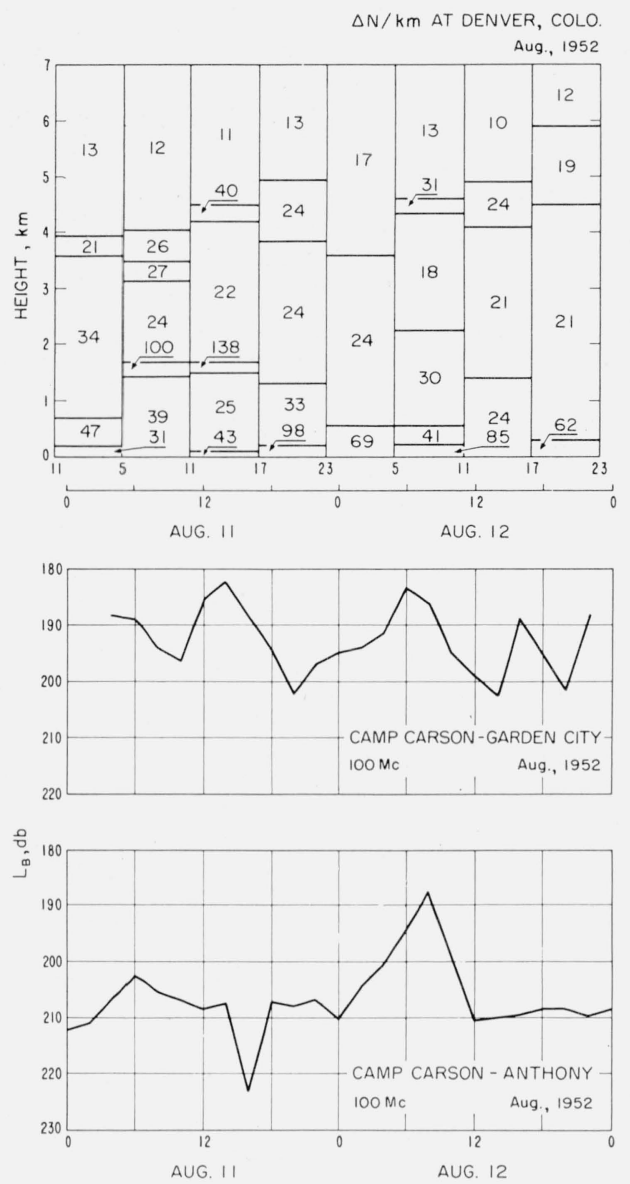

Figure 11. Comparison of transmission loss with the gradient of refractive index of the atmosphere.

cause of remarkable diurnal variations in the lower atmosphere. It is felt that the lowest atmosphere occasionally plays a role, probably by superrefraction, but is not frequent enough to determine the general characteristics of the variation in transmission loss. A study reported elsewhere [13] shows a similar result on the contribution of elevated layers and also of superrefractive layers, based upon experimental data obtained on an overland propagation path. Considerations of the lowest atmosphere should be made in further studies to distinguish its effects from those of the upper atmosphere.

\section{Suggestions as to the Probable Impor- tance of Radiometeorological Factors on Beyond-Horizon Communications}

Preparing for the further studies on prediction of variations in transmission loss within a month, we tried to disclose dominant radiometeorological factors in propagation beyond the horizon. Near the horizon, where the diffraction mode is considered predominant, the gradient of refractive index at the earth's surface may largely affect the received field strength. However, effects of elevated layers seem to increase rapidly just beyond the horizon, emphasized by refraction in the lowest part of the atmosphere. In this region, diurnal variation in field strength may occur on an overland path corresponding to the variation in the atmosphere. Diurnal variation decreases as the crossover height increases, and diminishes at crossover heights higher than about $500 \mathrm{~m}$ above the ground. In propagation far beyond the horizon, no significant diurnal characteristic is found. At such ranges, the received field is mainly controlled by discontinuity layers that are dependent on airmass properties and may be occasionally affected by superrefraction.

This interpretation, which is not based upon a suificient amount of data to give a final conclusion, may suggest, perhaps, that the effects of radiometeorological factors in the common volume play the most important role in communications beyond the horizon. It depends, of course, upon the property of propagation path, geographic situation, season, and climatological condition. Nevertheless, the atmospheric refractive index structure within the common volume looks like the most promising factor which determines the general characteristics of variations in transmission loss within a month, from the standpoint of practical communications. Perhaps a correction due to refraction may be necessary just beyond the horizon.

This interpretation would also be helpful for better understanding of the description of the seasonal variation in transmission loss in terms of surface refractivity or $N$-gradient at the earth's surface.

\section{Conclusion}

Propagation characteristics beyond the horizon should be discussed in two categories. In the first case, when crossover height is located in the diurnally varying portion of the atmosphere, diurnal variation in field strength should be taken into consideration. In the second case, when it is higher than about $500 \mathrm{~m}$ above the ground, field strength is largely dependent on the refractive index structure of the atmosphere free from the earth's surface. In both cases, the most dominant radiometeorological factor is considered to be the laminar structure of the atmosphere, which might be utilized for the expression of variations in transmission loss within a month.

The author thanks the United Nations which made possible his study in Boulder Laboratories, NBS, and the Federal Communication Commission, and National Bureau of Standards, Department of Commerce, without whose arrangements of his study program this paper could not have been written.

The author expresses his particular appreciation to B. R. Bean for his helpful advice and criticism as well as for his efforts in arranging radio and meteorological data obtained by NBS, and to H. B. Janes 
for his valuable information and advice in the analysis of diurnal variation. He also thanks the many individuals in the Radio Propagation Engineering Division, Boulder Laboratories, NBS, and in the Radio Wave Propagation Section, ECL, N.T. \& T., who contributed to the propagation experiments and to the reduction of the obtained data.

\section{References}

[1] B. R. Bean and F. M. Meaney, Some applications of the monthly median refractivity gradient in tropo spheric propagation, Proc. IRE 43, 1419 (1955).

[2] K. A. Norton, P. L. Rice, and L. E. Vogler, The use of angular distance in estimating transmission loss and fading range for propagation through a turbulent atmosphere over irregular terrain, Proc. IRE 43, 1489 (1955).

[3] R. E. Gray, The refractive index of the atmosphere as a factor in tropospheric propagation far beyond the horizon, IRE Natl. Conv. Record (1957).

[4] M. Onoe, M. Hirai, and S. Niwa, Results of experiment of long-distance overland propagation of ultrashort waves, J. Radio Research Labs., Japan 5, 79 (1958).

[5] H. G. Booker and W. E. Gordon, A theory of radio scattering in the troposphere, Proc. IRE 38, 401 (1950).
[6] T. J. Carroll and R. M. Ring, Propagation of short radio waves in a normally stratified troposphere, Proc. IRE 43, 1384 (1955).

[7] O. Tukizi, Diffraction theory of tropospheric propagation near and beyond the radio horizon, IRE, Trans. AP-7, 261 (1959).

[8] H. T. Friis, A. B. Crawford, and D. C. Hogg, A reflection theory for propagation beyond the horizon, B.S.T.J. $\mathbf{3 6 ,} 627$ (1957).

[9] H. B. Janes, An analysis of within-the-hour fading in 100-to-1,000-Mc transmissions, J. Research NBS 54, 231 (1955).

[10] P. A. Sheppard, The structure and refractive index of the lower atmosphere, Meteorological factors in radiowave propagation, Conf. Rept. at Roy. Inst., London, England, p. 37 (1946).

[11] F. Ikegami, M. Haga, T. Fukada, and H. Yoshida, Observations of ducts in the lower atmosphere and their effects on microwave fading (in Japanese), Elec. Commun. Lab., NT \& T Corp., Tokyo, Japan, rept. No. 910 (1956).

[12] T. Fukami, S. Ieiri, F. Ikegami, and H. Fujimura, Beyond-horizon propagation characteristics over sea (No. 1) (in Japanese) Elec. Commun. Lab. Tech. J., Tokyo, Japan, 8, No. 6 (1959).

[13] B. R. Bean, Sur l' utilisation des observations méteorologiques courantes en propagation radio électrique, L' Onde Elec. 362, 411 (1957).

Boulder, Colo.

(Paper 64D3-53) 